P0105

\title{
EXTRAPOLATION OF PHOSPHOR CONVERTED WHITE LED SPECTRA BEYOND THE VISIBLE WAVELENGTH
} RANGE

\author{
Tobias Schneider et al.
}

DOI 10.25039/x46.2019.PO105

from

CIE x046:2019

Proceedings

of the

29th CIE SESSION

Washington D.C., USA, June 14 - 22, 2019

(DOI 10.25039/x46.2019)

The paper has been presented at the 29th CIE Session, Washington D.C., USA, June 14-22, 2019. It has not been peer-reviewed by CIE.

(c) CIE 2019

All rights reserved. Unless otherwise specified, no part of this publication may be reproduced or utilized in any form or by any means, electronic or mechanical, including photocopying and microfilm, without permission in writing from CIE Central Bureau at the address below. Any mention of organizations or products does not imply endorsement by the CIE.

This paper is made available open access for individual use. However, in all other cases all rights are reserved unless explicit permission is sought from and given by the CIE.

CIE Central Bureau

Babenbergerstrasse 9

A-1010 Vienna

Austria

Tel.: +4317143187

e-mail: ciecb@cie.co.at

www.cie.co.at 


\title{
EXTRAPOLATION OF PHOSPHOR CONVERTED WHITE LED SPECTRA BEYOND THE VISIBLE WAVELENGTH RANGE
}

\author{
Schneider, T. ${ }^{1}$, Dekker, P. ${ }^{2}$, Young, R. ${ }^{1}$, Blattner, P. ${ }^{3}$, Poikonen, T. ${ }^{4}$ \\ 1 Instrument Systems GmbH, Munich, GERMANY , ${ }^{2}$ VSL, Delft, NETHERLANDS, ${ }^{3}$ METAS, Bern, \\ SWITZERLAND, ${ }^{4}$ VTT, Espoo, FINLAND \\ t.schneider@instrumentsystems.com
}

DOI 10.25039/x46.2019.PO105

\begin{abstract}
CIE TC 2-90 is currently working to propose a reference spectrum for use in photometer calibrations as a complement to CIE illuminant A, based on the white LED illuminant LED-B3 recently published by CIE as part of CIE 015: Colorimetry, $4^{\text {th }}$ edition (CIE 2018). Calibrating photometers with sources realizing LED-B3 is expected to reduce spectral mismatch errors in photometric measurements for a wide range of light sources and will alleviate the dependence on halogen lamps for photometric calibrations.

In order to compare source spectra realizing LED-B3 to equivalents over the full wavelength range of radiation emission of the source, an extrapolation method for LED-B3 beyond the wavelength range of its current definition $(380 \mathrm{~nm}$ to $780 \mathrm{~nm}$ ) will have to be defined.

We present models for the extrapolation of phosphor converted white LED spectra beyond the visible wavelength range and apply them to LED-B3 as well as measured spectra of white LED products.
\end{abstract}

Keywords: Photometry, Colorimetry, LED, spectrum, model, parametrization, illuminant

\section{Introduction}

White LEDs are progressively replacing incandescent lamps as predominant light sources in general lighting applications. This rapid development leads to novel demands and challenges from fundamental metrology over lighting design to legislative regulations and standardization.

On the metrological side, incandescent sources realizing CIE standard illuminant A (CIE2014) are currently the standard for photometer calibration and CIE standard illuminant $A$ is part of the definition of most quality indices characterizing the performance of photometers. With the diminishing availability of high quality incandescent calibration sources, other types of calibration sources will have to be introduced eventually. At the same time, photometric measurements become in general less accurate if the light source used for photometer calibration (illuminant $A$ ) has a spectral power distribution significantly different from that of the light source to be measured (often a white LED), because of spectral mismatch between the employed optical $V_{\lambda}$-filters and the $V_{\lambda}$-curve defined by CIE.

To help address those issues and provide guidance for future developments in lighting, the $\mathrm{CIE}$ has named "New Calibration Sources and Illuminants for Photometry, Colorimetry, and Radiometry" as one of the ten top priority topics in its 2016 research strategy (http://cie.co.at/research-strategy). As a first step, CIE has included a list of 10 reference LED spectra in the appendices of the $4^{\text {th }}$ edition of its technical report on Colorimetry (CIE2018) and formed technical committee TC2-90 (LED Reference Spectrum for Photometer Calibration) which will evaluate one of the LED reference spectra from (CIE2018) introduced originally in (Kokka2018), LED-B3, as the preferred candidate for complementing CIE Illuminant $A$ in photometer calibration.

Illuminant LED-B3 is defined in (CIE2018) from $380 \mathrm{~nm}$ to $780 \mathrm{~nm}$ in $5 \mathrm{~nm}$ steps. While this wavelength range and sampling is in general sufficient for most colorimetric or photometric calculations, physical realizations of LED-B3 will potentially have non-negligible emission of 
radiation outside this wavelength range. In order to allow for a physically meaningful and mathematically rigorous comparison of LED-B3 to any of its physical realizations over the full emission wavelength range of the physical source, an extrapolation as well as an interpolation strategy for LED-B3 beyond the wavelengths its definition is needed. The CIE research strategy also requests a description of potential new references over the wavelength range from $360 \mathrm{~nm}$ to $830 \mathrm{~nm}$.

In the following we discuss potential extrapolation strategies for LED-B3 beyond the wavelength range of $380 \mathrm{~nm}$ to $780 \mathrm{~nm}$, test them on measured spectra of white LEDs and apply them to LED-B3.

\section{Models for the photo-emission of phosphor converted white LEDs}

Passing the radiation emitted from a blue LED through a layer of a suitable phosphor material that absorbs part of the radiation and has an emission band centred in the yellow or red spectral region leads to a spectral power distribution that is perceived as white light by humans (see Fig.1).

In the simple case that the emission of the phosphor has negligible spectral overlap with the emission of the blue LED, a model of the spectral emission of a phosphor converted white LED can describe the parts of the emission spectrum of the white LED related to emission processes of the blue LED and the parts related to conversion of the blue light by the phosphor material independently.

For most phosphor converted white LEDs, the emissions of blue LED and phosphor have significant spectral overlap which would require a more complicated model that calculates each component and hence can simulate the combined spectrum.

However, in the spectral regions of interest for extrapolation of spectral data around $380 \mathrm{~nm}$ and $780 \mathrm{~nm}$, the spectral overlap of blue LED and phosphor emission is negligible and the wings of the spectral power distribution can to good approximation be treated as in the more simple case of no spectral overlap.

Illuminant LED-B3 is constructed as a superposition of spectral power distributions derived from measurement data of several physical sources with similar correlated colour temperatures in order to be representative of the class of phosphor converted white LEDs with a correlated colour temperature of $4100 \mathrm{~K}$ without matching exactly the spectral power distribution of any particular existing LED product. Therefore, the spectral power distribution defined by LED-B3 does not necessarily need to conform to any physical model. As we will show below, models of the spectral power distribution based on models of the physics of phosphor converted white LEDs and tested on measurement data of real devices also describe the spectral distribution of LED-B3 in the wavelength ranges of interest for spectral extrapolation with high accuracy.

Fig.1 below shows illuminant LED-B3 and the results of fits to the blue LED peak and phosphor peak respectively using models discussed in the following sections. 


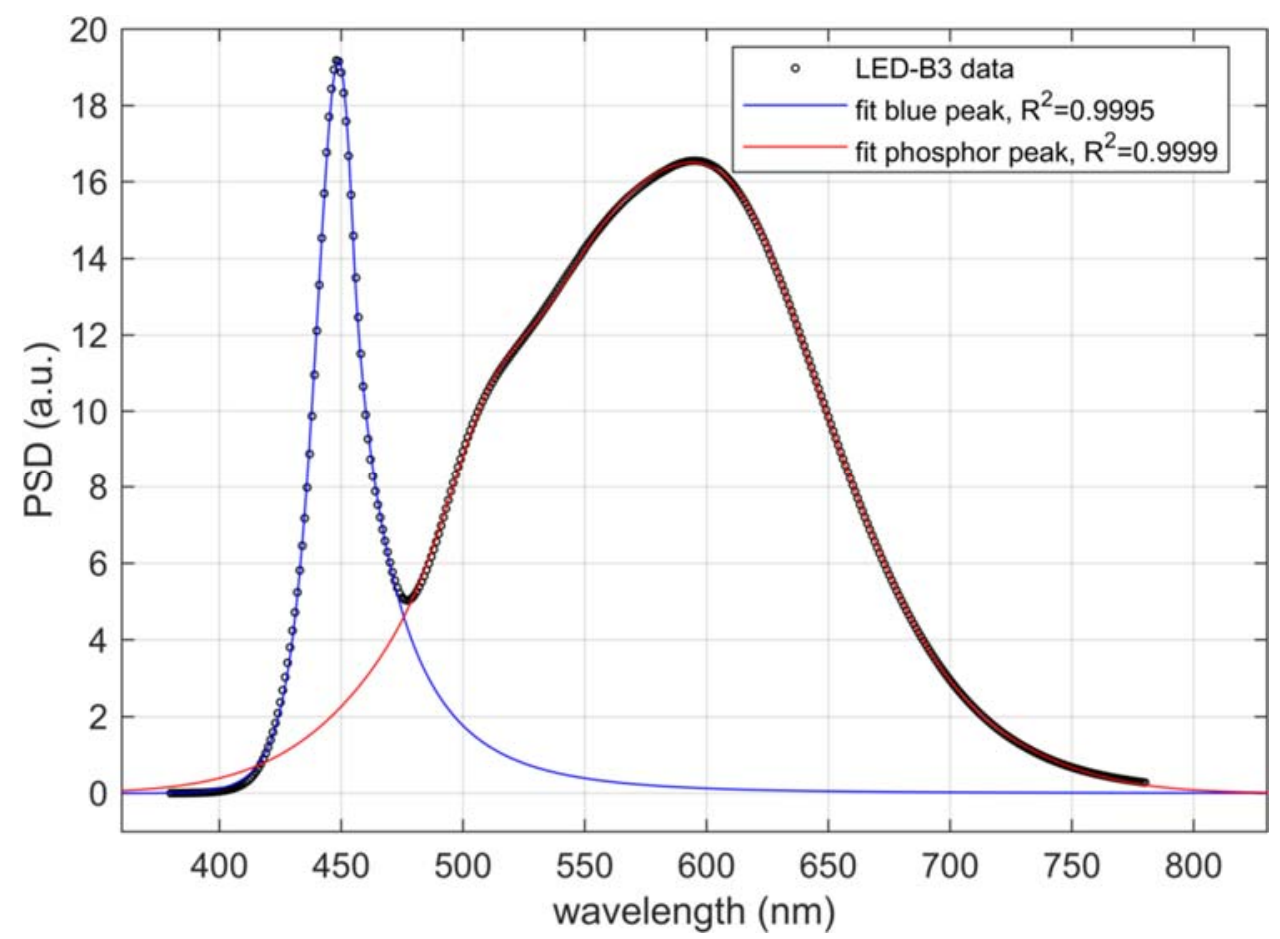

Figure 1 - Illuminant LED-B3 and fit curves for the blue peak (see section 2.1) and the phosphor peak (see section 2.2). $R^{2}$ is the coefficient of determination for the fit.

\subsection{Blue LED emission}

The photo-emission of single colour LEDs based on semiconductor materials is well studied and reliable models for their photo-emission are available (Katahara2014, Schneider2017, Vaskuri2015, Vaskuri2018). These models predict that the spectral power distribution of LEDs follows a simple exponential decay for wavelengths well below the maximum of the distribution:

$$
\Phi_{L E D}(\lambda) \stackrel{\lambda_{0}-\lambda>\frac{w_{0}}{2}}{\longrightarrow} A \cdot \exp \left(-\frac{\left(\lambda_{0}-\lambda\right)}{w}\right)
$$

where $\lambda_{0}$ is the wavelength at the maximum of the spectral distribution, $w_{0}$ is the FWHM width of the spectral power distribution, $A$ is an amplitude that depends on the normalization of the spectral power distribution and $w$ is a characteristic decay constant which depends on the physics of the device. The physics behind the exponential decay of the spectral distribution at short wavelength (high energies) is the exponential decrease of the occupation difference of conduction and valence band of the semiconductor with increasing energy of the states, which is described to very good approximation by the Boltzmann approximation of the Fermi-Dirac distribution (Katahara2014).

For use in extrapolation of LED-B3, or any other spectral distribution, the parameters of equ.(1) can be determined from a fit of equ.(1) to the data. Extrapolation is then done by calculating equ.(1) at any wavelength of interest. The quality of the extrapolation will depend on how well equ.(1) fits the data, how much data fulfilling the condition $\lambda_{0}-\lambda>\frac{w_{0}}{2}$ is available and (for experimental data) also on the signal-to-noise ratio of the data.

If the spectral distribution to be extrapolated closely follows equ.(1) for all data points, and the data has a good signal-to-noise ratio, the extrapolation can be simplified to a linear extrapolation of the natural logarithm of the spectral distribution $\Phi(\lambda)$ (logarithmic extrapolation):

$$
\log (\Phi(\lambda))=\log \left(\Phi\left(\lambda_{N}\right)\right)+\frac{\log \left(\Phi\left(\lambda_{N}\right)\right)-\log \left(\Phi\left(\lambda_{N-1}\right)\right)}{\lambda_{N}-\lambda_{N-1}}\left(\lambda-\lambda_{N}\right)
$$


where $\lambda_{N}$ and $\lambda_{N-1}$ are the wavelengths of the two data points used for extrapolation (if $\lambda_{N}<$ $\lambda<\lambda_{N-1}$ equ.(2) describes linear interpolation).

An advantage of logarithmic extrapolation is that it requires only few data points and automatically guarantees continuity of the data. A fit of equ.(1) to the data will in general not match the fitted data exactly, which will introduce some differences of the extrapolation with respect to the data. The disadvantage of logarithmic extrapolation is that it is more susceptible to noise in the data and systematic deviations of individual data points from the functional form of equ.(1) and its accuracy in general decreases with distance to the last data point.

\subsection{Phosphor emission}

Radiation absorbed by the phosphor material excites the photoactive component of the material to excited electronic (and vibrational) states. The excited states decay mostly nonradiatively by internal conversion or inter-system crossing to sets of lower lying states which then can decay again radiatively. As a result, the phosphor converts the absorbed light of the blue LED to broadband emission at longer wavelength. The wavelength of maximum emission and the spectral distribution of the emission are, for a given spectral distribution of the emission of the blue LED, characteristic for the phosphor material, temperature and optical design of the LED.

The whole process of absorption, non-radiative transfer and emission is in general too complex to be described analytically even if the relevant physical properties of the phosphor material are known. Nevertheless, based on the general properties of the physical processes an approximation to the general functional shape of the spectral distribution of phosphor emission can be derived:

For the purpose of interaction with optical radiation, the phosphor can be described as a (potentially very large) set of quantum mechanical states. Neglecting the thermal motion of the atoms/molecules in the phosphor, the temporal dynamics of the interaction of this set of quantum states with the radiation field can be described by the multi-level generalization of the Maxwell-Bloch equations (ARECCHI1965). The interaction of the phosphor with radiation emitted by an incoherent source like an LED will be mostly also incoherent. Furthermore, nonlinear processes like multi-photon absorption or nonlinear frequency mixing will not play an important role at the light intensities emitted by the blue LED. In this case, the MaxwellBloch equations will reduce to a set of $1^{\text {st }}$ order linear rate equations for the population dynamics of the internal quantum states of the phosphor. Rate equations have been successfully used to model the emission of real phosphors (DU2017). The general solution for the population of each state will then be a simple exponential decay with an individual time constant and amplitude. The corresponding emission spectrum of the phosphor in the frequency domain is then by Fourier transform a superposition of Cauchy-Lorentz distributions. At the typical operation temperatures of the phosphor in a white LED, inhomogeneous Doppler broadening of the emission due to thermal motion of the emitting particles will transform the superposition of Cauchy-Lorentz distributions to a superposition of gaussian distributions:

$$
\Phi_{\text {phosphor }}(\lambda) \cong \sum_{i=1}^{N} c_{i} \cdot G_{i}(\lambda) ; \quad G_{i}(\lambda)=\exp \left(-\frac{\left(\lambda-\lambda_{i}\right)^{2}}{w_{i}^{2}}\right)
$$

where the coefficients $c_{\mathrm{i}}$ and $w_{\mathrm{i}}$ contain the information about the details of the physics of the system.

As for the description of the LED emission discussed above, equation (3) gives the general structure of a fit-function suitable to match the spectral shape of the phosphor emission.

The principal number of terms $N$ in equation (3) is equal to the number of radiatively decaying electronic states of the phosphor materials counting also all potential vibrational sub-states of each electronic state. $N$ will therefore in general be very large. However, a superposition of large number of Gaussian distributions with typical separations of adjacent mean values less 
than the width of the distributions will again look similar to a superposition of $M \ll N$ Gaussian distributions (with different coefficients $c_{\mathrm{j}}$ and $w_{\mathrm{j}}$ ), such that

$$
\sum_{i=1}^{N} c_{i} \cdot G_{i}(\lambda) \cong \sum_{j=1}^{M \ll N} \tilde{c}_{j} \cdot \tilde{G}_{j}(\lambda)
$$

Applying equation (4) to measured data shows that for a good fit of the long wavelength tails of the phosphor emission of white LEDs, $M=3$ is usually sufficient (see below). Other studies not reported here show that for a good fit of the full LED-B3 spectrum $M \sim 8$ is often sufficient to accurately model the spectral shape of the phosphor peak (see Fig.1).

\section{Application to measurement data}
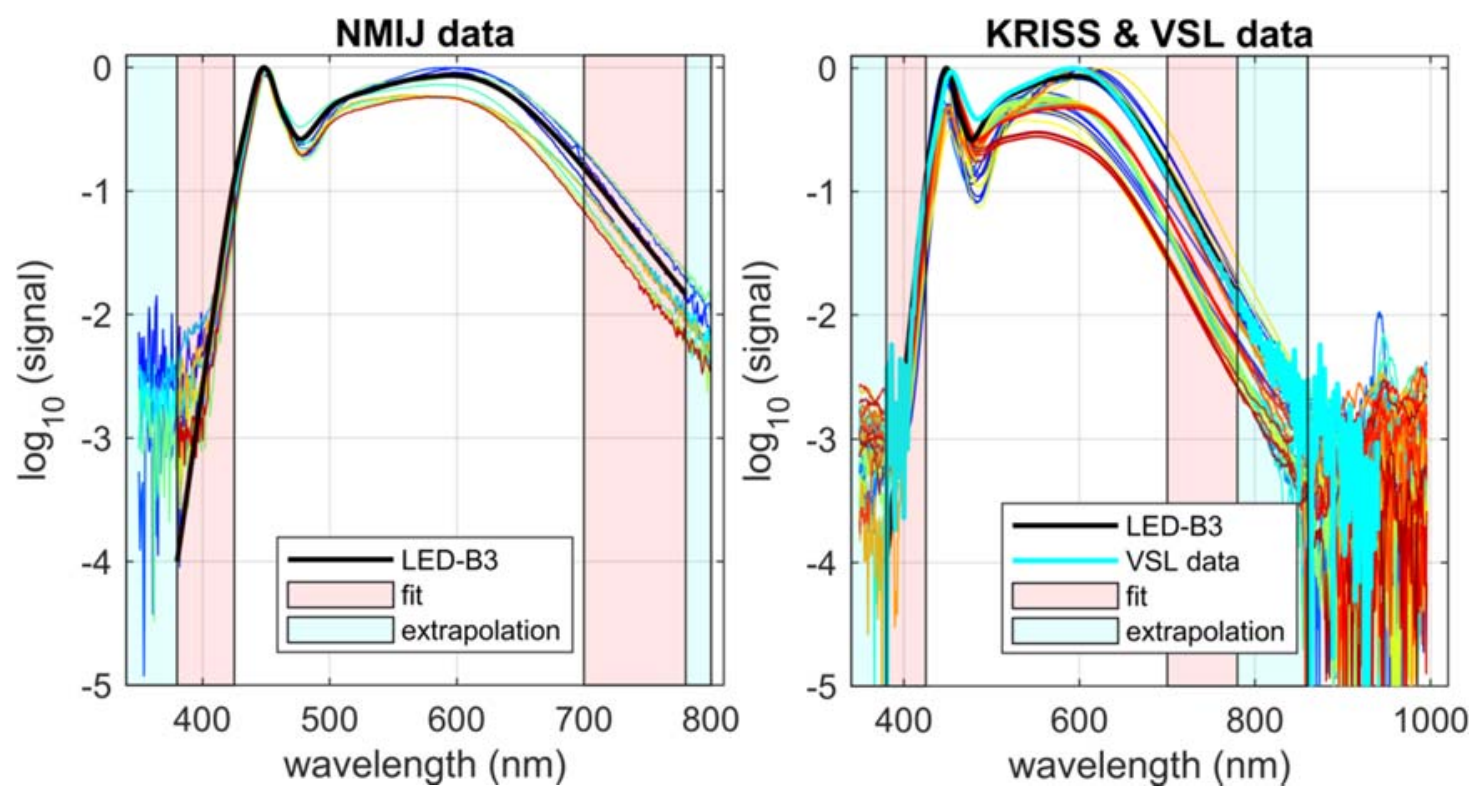

Figure 2 - Test data sets measured at NMIJ (left) and KRISS \& VSL (right). Red shaded areas indicate the data used for fitting. Blue shaded areas mark the wavelength ranges for comparison of data with extrapolation using the fit models.

Tests of the models derived in the previous section were done on three sets of LED data with a wide range of CCT values (Fig.2): 15 spectra measured at NMIJ (National Metrology Institute of Japan), 45 spectra measured at KRISS (Korea Research Institute of Standards and Science) and one spectrum measured at VSL (Dutch National Metrology Institute). While all spectra analysed represent high quality measurements, the data shows typical properties of real world data like noise, finite dynamic range and sometimes systematic effects like stray light.

For all data sets, a fit of equ.(1) to the data was performed in the wavelength range from 380 $\mathrm{nm}$ to $420 \mathrm{~nm}$. At long wavelengths a fit of equ.(3) with $N=3$ was applied to data in the wavelength range from $700 \mathrm{~nm}$ to $780 \mathrm{~nm}$. Fig. 3 shows that the overall quality of fit does not improve significantly for $N>2$, but adding an additional term does not increase the complexity of the fit procedure much while still improving the fit for some data sets. The fits were then used to extrapolate the data to wavelengths below $380 \mathrm{~nm}$ and above $780 \mathrm{~nm}$ and the results were compared to the original data (Fig.4). 

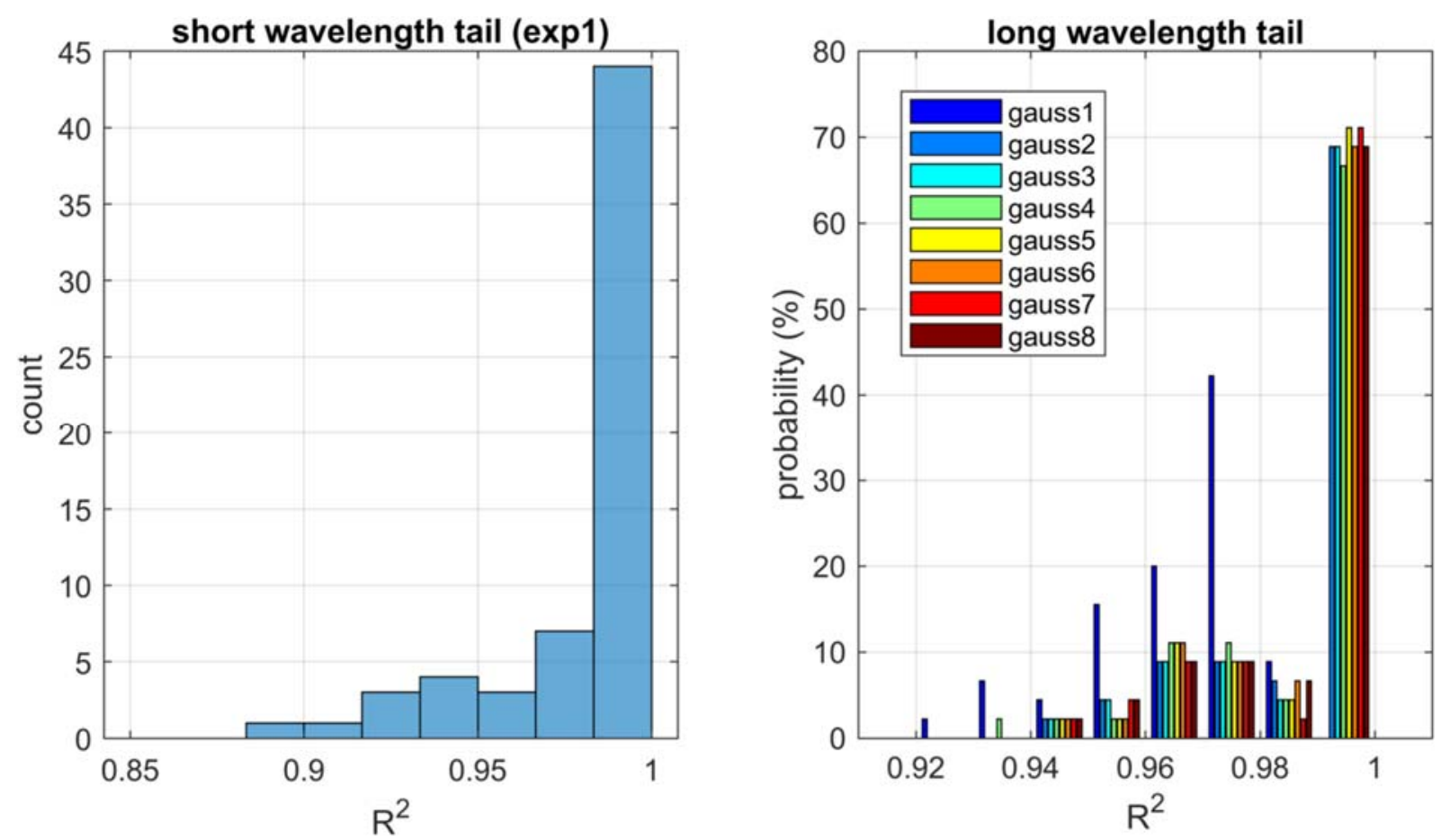

Figure 3 - Performance of the fit models from section2. Left: Statistics of the coefficient of determination $R^{2}$ for a fit of equ.(1) to the data from $380 \mathrm{~nm}$ to $420 \mathrm{~nm}$. Right: Statistics of the coefficient of determination $R^{2}$ for a fit of equ.(2) to the data from $700 \mathrm{~nm}$ to $780 \mathrm{~nm}$ for different numbers of terms $N$.

Since the power spectral density is already close to zero in parts of the extrapolation ranges and noise in the data becomes relevant, looking at the absolute magnitudes of the differences of data and extrapolation is not very instructive. Fig. 4 shows a statistical analysis in the form of probability plots for the data and the fit residuals in the extrapolation range (calculated as fit - data) corrected for their respective mean values.
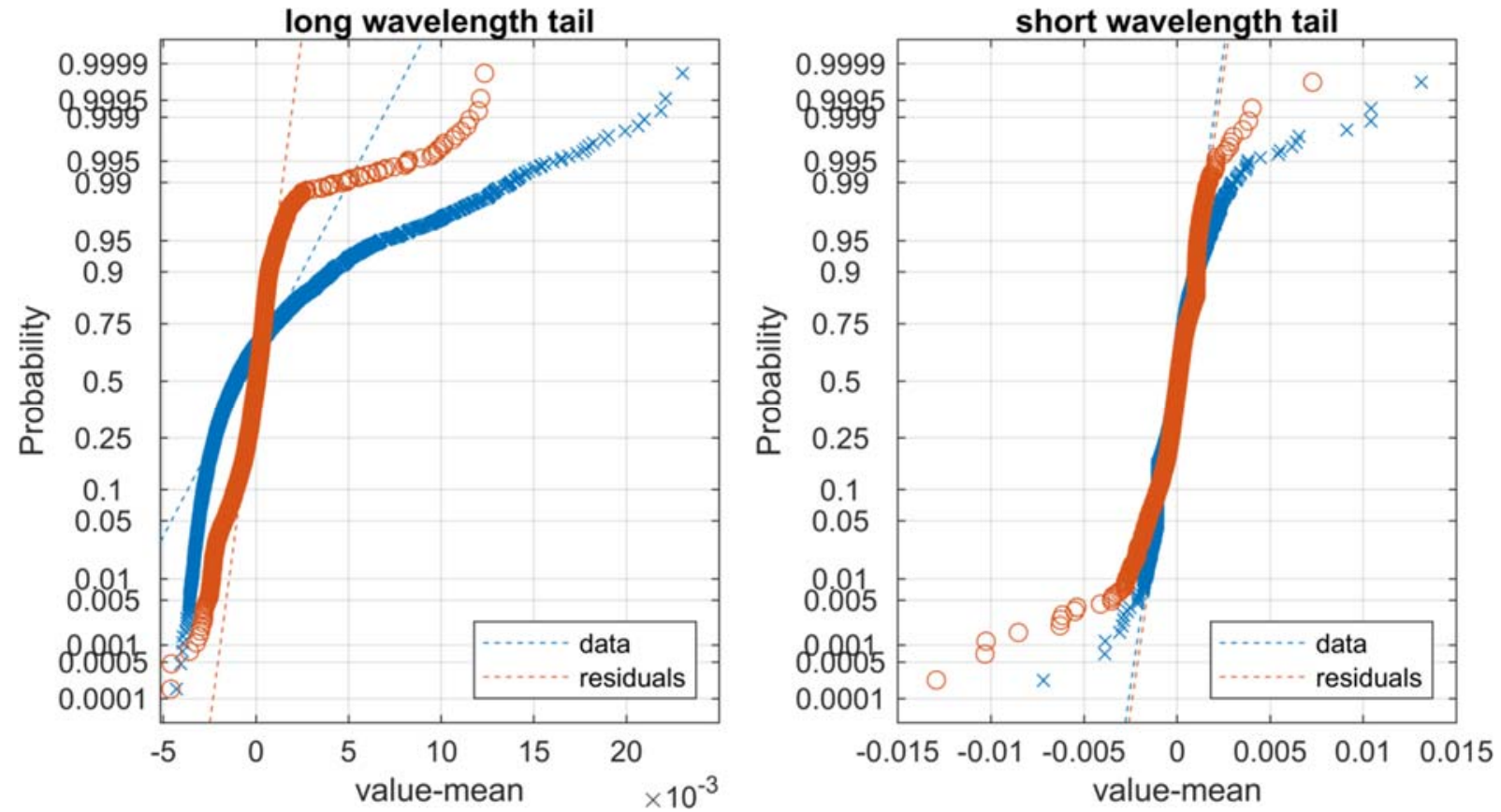

Figure 4 - Probability plots for data and fit residuals corrected for their respective mean values in the wavelength ranges of extrapolation. 
At short wavelengths, the spectral amplitudes below $380 \mathrm{~nm}$ are very small and noise dominates the signals. The distribution of the data is therefore close to a normal distribution. The distribution of residuals of the extrapolation is even closer to a normal distribution for positive values and is also narrower, indicating that the extrapolation matches the data in general well. At negative values the distribution of the residuals is wider than that of the data and deviates more from a normal distribution. One reason is that equ.(1) is always positive, while the data can also take negative values because of noise and dark signal subtraction. In addition, some data sets show indication of a systematic increase of the spectral amplitude towards lower wavelength, probably caused by small amounts of stray light in the measurement systems. Again this systematic is not modelled by equ.(1), leading to an additional small excess of negative residuals.

At long wavelengths, the spectral amplitudes are still well above the noise over most of the extrapolation wavelength range, which leads to a strong deviation of the distribution from a normal distribution in Fig.4. The wavelength range for comparison was limited to wavelengths below $860 \mathrm{~nm}$. At wavelengths above, the data becomes dominated by noise and systematic measurement artefacts, which makes a meaningful comparison to the fit results difficult. The distribution of the residuals is much closer to normal except at the outer wings of the distribution and narrower than the data distribution at all values. Again this shows that the extrapolation by the fits matches the data in general quite well.

The spectrum measured at VSL is quite close to LED-B3 and has very good signal to noise ratio at wavelengths above $780 \mathrm{~nm}$. Fig. 5 shows an analysis of the fit residuals. At short wavelengths, the quality of extrapolation by the fit models cannot be verified since there is not sufficient data below $380 \mathrm{~nm}$. At wavelength below $420 \mathrm{~nm}$, the fit of equ.(1) to the data matches the data very well down to the level where the signal is dominated by noise.

In this case, the extrapolation will always be good, because the signal should essentially be zero in the extrapolation range. At wavelength above $780 \mathrm{~nm}$, the fit of equ.(3) with $N=3$ applied at wavelengths of $700 \mathrm{~nm}$ to $780 \mathrm{~nm}$ gives good prediction of the data at longer wavelengths except in the range from $800 \mathrm{~nm}$ to $850 \mathrm{~nm}$ where the data is systematically underestimated.
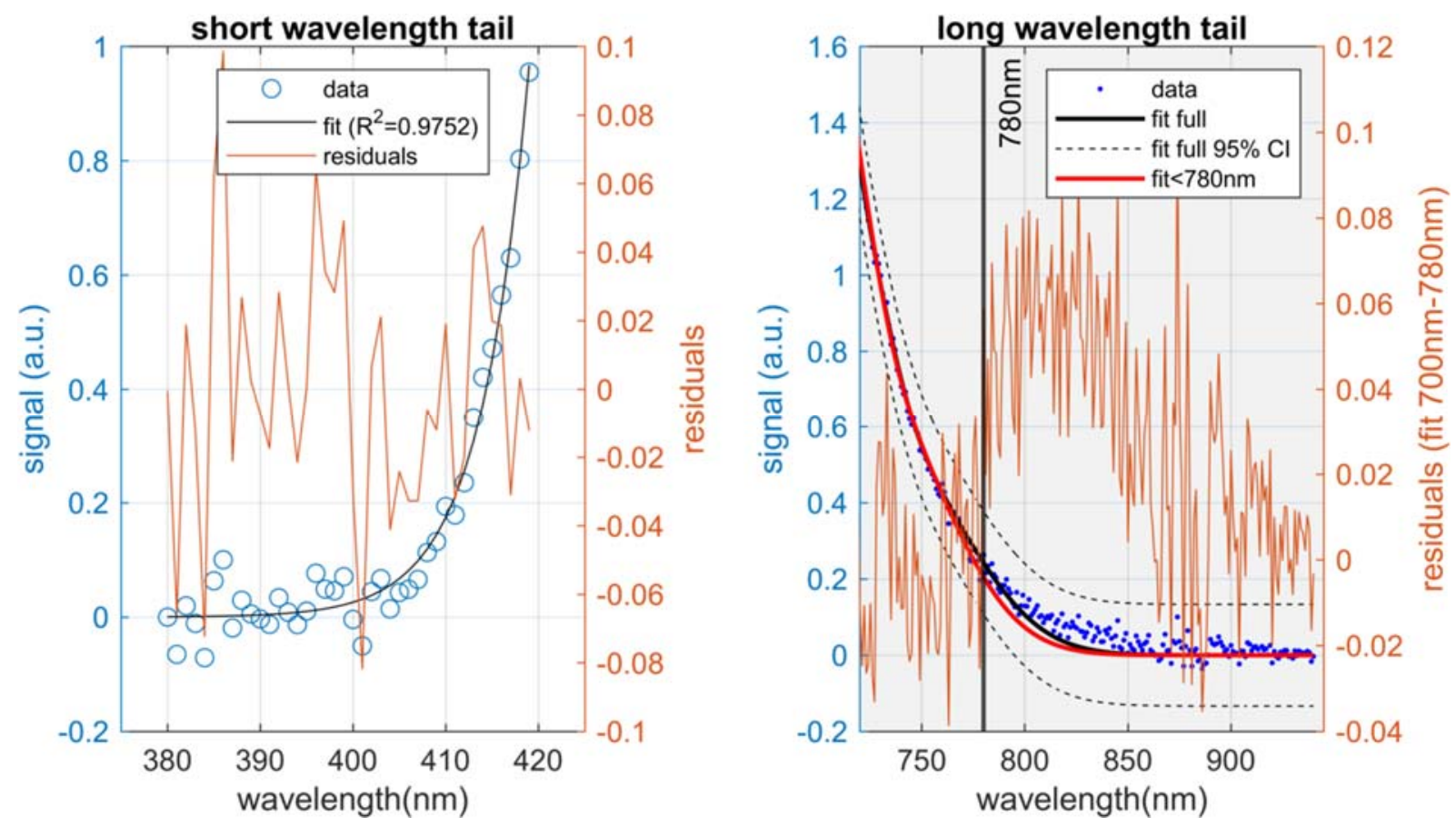

Figure 5 - Fit and extrapolation results for the VSL data. Left: fit of equ.(1) to data at wavelengths below $420 \mathrm{~nm}$. Right: fit of equ.(3) with $\mathrm{N}=3$ to data in the wavelength range from $700 \mathrm{~nm}$ to $780 \mathrm{~nm}$ (red) compared to a fit from $700 \mathrm{~nm}$ to $1000 \mathrm{~nm}$. 
A fit of the same model to the wavelength range from $700 \mathrm{~nm}$ to $1000 \mathrm{~nm}$ gives only a slight improvement and similar results at wavelengths below (above) $750 \mathrm{~nm}(850 \mathrm{~nm})$ indicating a systematic deviation of specific device behaviour from the model or a systematic measurement artefact. Also for this data set an extrapolation based on equ.(3) would give a physically meaningful extrapolation if there was no data beyond $780 \mathrm{~nm}$.

\section{Application to LED-B3}
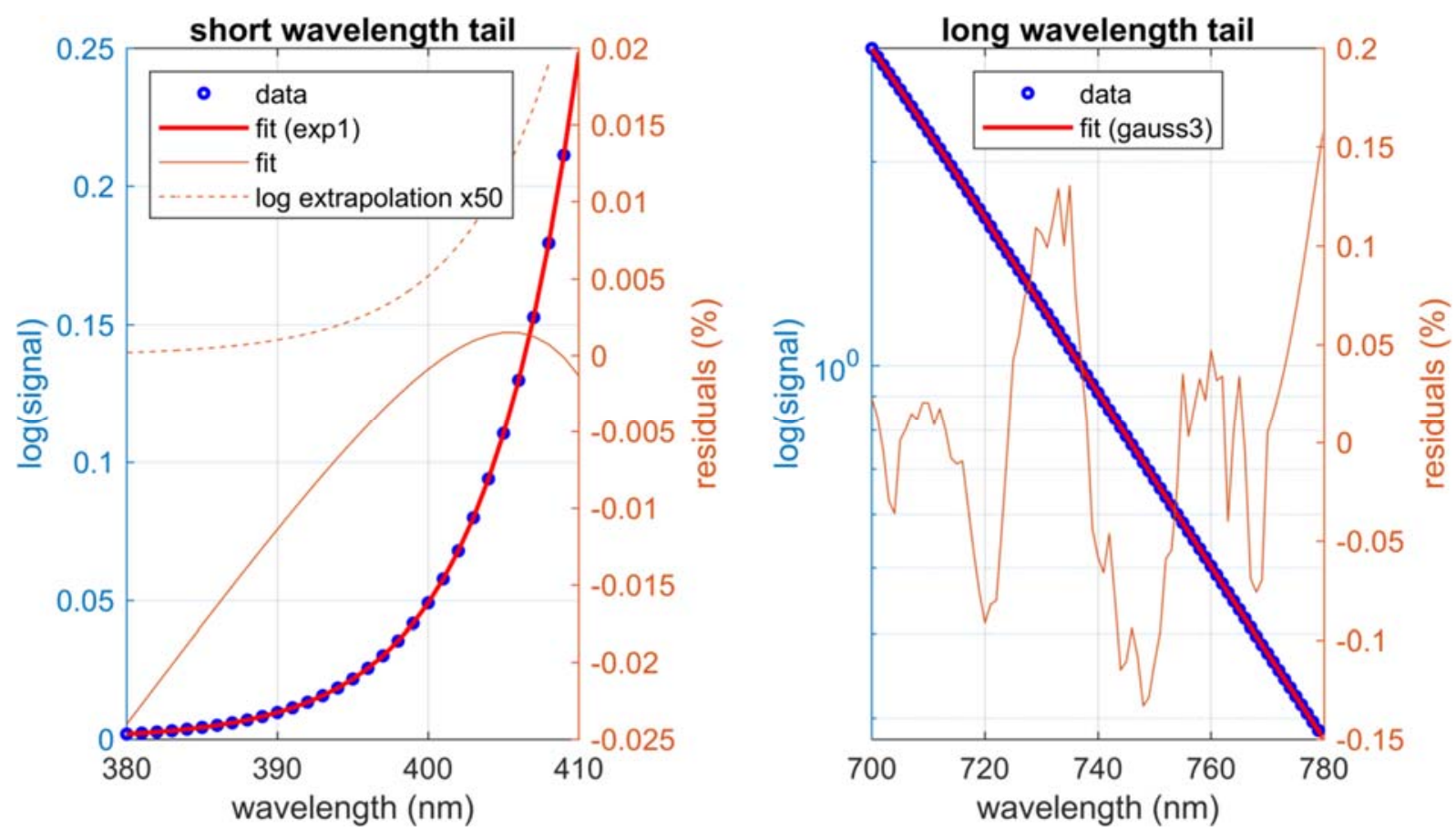

Figure 6 - Fits of equ.(1) (left) and equ.(3) with $N=3$ (right) to LED-B3.

Having confirmed that the models introduced in section2 for the spectral power distributions of phosphor converted white LEDs work well on measured data of actual LED products, we apply the models to LED-B3.

Fig.6 shows that the models equ.(1) and equ.(3) with $N=3$ fit the wings of the spectral distribution of LED-B3 very well $\left(R^{2}=1\right.$ in both cases $)$. At short wavelengths the quality of fit decreases slightly if the fit covers a larger wavelength range. The relative fit residual at $380 \mathrm{~nm}$ is only about $-0.02 \%$, so extrapolation using the fit will not introduce a significant discontinuity in the data. Because LED-B3 very closely follows equ.(1) at short wavelengths and has no noise, logarithmic extrapolation might be preferred to a fit of equ.(1) because of the even lower residuals in description of the data.

At wavelengths above $700 \mathrm{~nm}$ a fit of equ.(3) leads to larger relative residuals as the fit of equ.(1) at short wavelengths, but the value of $0.15 \%$ at $780 \mathrm{~nm}$ is small enough that extrapolation will not introduce significant discontinuity in the data. Again, the simple model equ.(3) fits the data very well, despite the fact that LED-B3 is an artificial spectrum.

\section{Conclusion and Outlook}

We presented two fit models suitable for the extrapolation of the spectral power distribution of illuminant LED-B3 from (CIE2018) for use as an LED reference spectrum for photometer calibration, as well as measured spectral power distributions of phosphor converted white LEDs with a wide range of correlated colour temperatures.

At wavelengths below $410 \mathrm{~nm}$ (to $420 \mathrm{~nm}$ ) the spectral power distributions are well described by a single exponential decay in accordance with the predictions of a priori models of the physics of single colour LEDs. 
At wavelengths above about $700 \mathrm{~nm}$ basic physical considerations of the interaction of the phosphor in the LED with the light from a blue LED predict that the spectral power distributions can be described by a superposition of $N$ Gaussian functions. Tests on measured data show that typically $N=3$ is sufficient for an accurate fit.

The models were validated with sets of measured data. Application to LED-B3 gives excellent fit results allowing continuous extrapolation of LED-B3 to any wavelength in a way that is consistent with the physics of real phosphor converted white LEDs.

The extrapolation models introduced in this paper are designated to be used in the definition of the final LED reference spectrum for calibration use by CIE TC2-90.

\section{Acknowledgements}

The authors acknowledge Seongchong Park and Dong-Hoon Lee from KRISS and Kenji Godo from NMIJ for providing measurement data for the analysis presented.

\section{References}

CIE 2018. CIE 015:2018. Colorimetry, $4^{\text {th }}$ Edition. Vienna: CIE.

CIE 2014. CIE S014-2/E:2006. Colorimetry - Part 2: CIE Standard Illuminants. Vienna: CIE.

KOKKA, A., ET AL., 2018. Development of white LED illuminants for colorimetry and recommendation of white LED reference spectrum for photometry. Metrologia, 55, 526534.

KATAHARA, J.K., HILLHOUSE, H.W., 2014. Quasi-Fermi level splitting and sub-band gap absorptivity from semiconductor photoluminescence, Journal of Applied Physics, 116, 173504.

SCHNEIDER, T., YOUNG, R., 2017. Analytical models for photo-emission spectra of single colour LEDs. CIE x044:2017, 694-703.

VASKURI, A., ET. AL., 2015. Modeling the spectral shape of InGaAIP-based red light-emitting diodes. J. Appl. Phys., 118, 203103.

VASKURI, A., ET. AL., 2018. Relationships between junction temperature, electroluminescence spectrum and ageing of light-emitting diodes. Metrologia, 55, S86.

ARECCHI, F., BONIFACIO, R., 1965. Theory of optical maser amplifiers. IEEE Journal of Quantum Electronics, 1 (4), 169-178

DU, K. 2017. The rate equation based optical model for phosphor-converted white lightemitting diodes. J. Phys. D: Appl. Phys., 50, 095101. 